\title{
The Thailand Declaration of the International College of Nutrition; Can Functional Foods and Functional Crops Decrease the Risk of Chronic Diseases and Provide Wellness?
}

\author{
Wiriya Phomkong 1, Ekasit Onsaard 1*, Dherapol Bansiddhi 1, Ram B. Singh 2, \\ Buncha Ooraikul ${ }^{3}$, Arunporn Itharat ${ }^{4}$, Fabien De Meester ${ }^{5}$, Jagdish P. Sharma ${ }^{2}$, Rana G. Singh ${ }^{6}$, \\ Usha Singh ${ }^{6}$, Harpal S. Buttar ${ }^{7}$ Istvan G. Telessy ${ }^{8}$, Toru Takahashi ${ }^{~}$, Lech Ozimek ${ }^{10}$, Jae-Kwan \\ Hwang ${ }^{11}$, Kohei Oda ${ }^{12}$ and Douglas W. Wilson ${ }^{13}$ \\ 1 Indigenous Food Research and Industrial Development Unit, Faculty of Agriculture, Ubonratchathani \\ University, Ubon Ratchathani, Thailand 34190; wiriya.p@ubu.ac.th, e.onsaard@gmail.com, dherapol.b@ubu.ac.th, \\ 2 Halberg Hospital and Research Institute, Moradabad, India; rbs@tsimtsoum.net \\ 3 Department of Agricultural, Food \& Nutritional Science, Faculty of Agriculture, Life \& Environmental \\ Sciences, University of Alberta, Edmonton, AB, Canada T6G 2P5; buncha.ooraikul@gmail.com \\ 4 Center of Excellence on Applied Thai Traditional Medicine Research, Faculty of Medicine, Thammasat \\ University, Pathumthani 12120 ; iarunporn@yahoo.com \\ 5 The Tsim Tsoum Institute Krakow, Poland; fdm@tsimtsoum.net \\ $6 \quad$ Institute of Medical Sciences, BHU, Varanasi, India; rgsingh@bhu.ac.in, usha_path@bhu.ac.in \\ 7 Harpal S. Buttar, Faculty of Medicine, University of Ottawa, Ottawa, Canada; hsbuttar@bell.net \\ 8 University of Pécs Medical School, Szigeti str 12, H-7624 Pécs, Hungary; telessyist@vnet.hu \\ 9 Graduate School of Human Environmental Medicie, Fukuoka, Japan; takahashi@fwu,ac.jp \\ 10 Department of Agricultural, Food and Nutritional Science; lozimek@ualberta.ca \\ 11 College of Lifescience and Technology, Yonsei University, Seoul, Korea; jkhwang@yonsei.ac.kr \\ 12 Kyoto Institute of Technology, Kyoto, Japan; bika@kit.ac.jp \\ 13 School of Medicine, Pharmacy and Health Sciences, Durham University, Durham, UK; \\ d.w.wilson@durham.ac.uk \\ * Correspondence: e.onsaard@gmail.com Tel.: +66-45-353-519
}

\begin{abstract}
There is evidence that optimal nutrition is fundamental to human health and in the prevention of non-communicable diseases (NCDs) later in adult life. The identification, production and consumption of functional foods worldwide can increase health benefits for all who can access and afford such foods subject to advice from nutritionists. Recent meetings organized by the health agencies, give a crucial opportunity to make nutrition, a central part of the post-2015 sustainable human and agricultural development agenda. The aim of discussions in these meetings was to provide functional crops and foods to achieve optimal health by prevention of NCDs. It is possible that these efforts might ensure that the goals and targets set in the agenda are adequate to address the many challenges of global undernutrition as well as obesity which are major risk factors of NCDs. In many developing and middle income countries, food security provided by the governments, in one sense understandably, gave least consideration to functional foods supply and the prevention of obesity and metabolic syndrome, resulting in to emergence of NCDs. The Thailand Declaration reiterates that commitments to eradicate hunger and undernutrition as well as over-nutrition, and to increase investments in effective interventions; designers foods and designers crops. However, in planning coherent policies, our past experience on rapidly absorbed, energy-rich processed foods should be taken in to account while developing sustainable food systems. The food industry should be educated to exploit the expertise of food scientists and health professionals in designing functional foods taking cognizance of manufacturing and processing. Similarly, agriculture scientists may be actively involved in educating farmers so as to grow cash
\end{abstract}


crops providing functional foods. The aim should be to achieve an increase in the availability of functional foods to an extent, or by a policy, by which such foods are available to poors, at affordable cost to prevent hunger and undernutrition and related diseases as well as NCDs. In addition our efforts might help in developing an international consensus on how to approach the development of new designer foods by farmers and food industry to produce low glycemic index foods. Such efforts may establish an international framework for the prevention of NCDs, so that human susceptibility to these diseases is substantially diminished.

Keywords: Diet: Functional foods: Western foods: Mediterranean foods: Feeding, Plant breeding

\section{Introduction}

Functional foods are characterized by high nutrients and low energy which can influence physical and mental performance as well as psychological behavior that are characteristics of total health, including cardiovascular health [1-3]. Functional foods are known to provide benefit due to presence of biomolecules by addressing some physiological mechanisms through metabolic, transport uptake, metabolomics action in the body including reactive oxygen species and epigenesis. The identification, production and consumption of functional foods worldwide can increase health benefits for all who can access and afford them subject to advice from nutritionists and health agencies [4]. It has been observed worldwide that poor nutrition during antenatal period and childhood, as well obesity during infancy, may be risk factors for cardiovascular diseases (CVDs); e.g. coronary artery disease (CAD), hypertension and stroke, and type 2 diabetes in later adult life [1-5]. Therefore, optimal nutrition during pregnancy, childhood and adolescents, appears to be fundamental to optimal human health and wellness and in the prevention of NCDs later in adult life [1-6]. According to new estimates from the UN Food and Agriculture Organization (FAO), about 805 million people remain chronically undernourished, which may be the major underlying cause of death in this age group, accounting for $45 \%$ of child deaths worldwide [7-9]. Apart from this burden, more than 2 billion people appear to have deficiencies of nutrients; such as iodine, vitamin A, D, zinc, iron, omega-3 fatty acids, flavonoids and other antioxidants as well as essential and nonessential amino acids [1-9]. It is important to realize that chronic nutritional deficiency followed by optimal or high intake of energy rich foods do not allow conservation mechanisms of our body to adapt, resulting in obesity and metabolic syndrome later in adult life. This declaration serves to indicate the global extent of undernutrition; introduces briefly the food and farming transition which has occurred in more recent times of human development. The main objective is to discuss from food security in Asian context, indicating potential role of functional foods known for prevention of diseases and for health promotion. The aims of most agencies are to provide low glycemic index functional foods and functional farming outcomes that are rich in nutrients. It is possible that these efforts might ensure that the goals and targets set are adequate to address the many challenges of global malnutrition; including undernutrition, as well as obesity, which are major risk factors of NCDs. In addition our efforts might help in developing an international consensus on what foods should be provided to approach the prevention of malnutrition; to establish an international framework for the prevention of NCDs. It is important to provide food security to prevent global malnutrition and plan functional food security to pregnant mothers, infants and children for primordial prevention of NCDs because the time has come to consider 'feeding the world' sustainably [10]. Recently, the Sofia Declaration also proposed to health professionals and general population, particularly mothers, to learn about functional foods as well as Mediterranean-style diets such as whole grain, vegetables, fruits, eggs, fish, nuts and olive oil. These diets should be introduced and promoted to existing patients, prospective outpatients, and their family members: concentrating interventions on pregnant mothers, infants and children as well as the elderly, particularly in middle and lower income countries where NCDs are rapidly emerging[11]. The Paleolithic Homo sapiens, hunter-gatherers and early peasants, had marked variations in food consumption patterns compared to Asian and Homo economicus Western populations [12-14] (Tables 1and 2). The foods consumed by the Hunter-gatherers were mainly those foods provided by nature, but foods consumed by the modern world are manufactured by the industry which has adverse effects on risk factors of cardiovascular diseases (CVDs) and other NCDs. The food consumption pattern during human evolution from a nutritional and agricultural view point, can be divided into four categories; Homo sapiens, Hunter-gatherers, peasant farmers and modern farmers which have different attributes of social classes, food intakes and behavior. The modern world has very few primitive hunter-gatherers today, but in developing countries like India, the population has mainly farmers 
and rapidly growing towns comprising increasing number of poor urban slum dwellers and a large affluent society $[1,3,4,12,15]$.

Although, CVDs and other chronic diseases have become major causes of deaths among affluent societies, urban slum dwellers and farmers continue to suffer more often due to communicable diseases [5-7]. This review elicits evidence to as to whether food and agriculture can play important role in the prevention of NCDs. We also discuss the role of various foods and methods of agriculture, in relation to nutrients, available during early Palaeolithic times, compared to modern foods and agriculture.

\section{Food and Farming Transition}

Man started farming in Mesopotamia, approximately 10,000 years ago which in turn led to unprecedented technical development, agriculture, industry and commerce. Our diet was based on an enormous variety of whole grains and wild plants prior to the agricultural revolution concerned with initial transition from Hunter-gatherers [1-3]. Farmers and Hunter-gatherers also had excellent health characteristics; enormous physical activity possibly with limited mental stress, because stress is a function of industrialization, urbanization and globalization. Alcoholism and tobacco intake are now important behavioral risk factors of CVDs and other NCDs. The foods available to early peasants were natural; whole grains, seeds, fruits, vegetables, leaves, roots, fish, meat and egg which have lower glycemic index. The foods manufactured by food industry in general are processed and preserved and have high energy and high glycemic index with poor nutrient density [1-3] (Tables 1and 2).

In the Western World, the quest for rapid growth of crops for greater yield of foods involved large scale use of fertilizers and biotechnology. Attention was also focused on refining and processing of foods, storing and distribution, and there was a relentless search for a better economic model [5-7]. Industrialization and urbanization caused economic development and affluence in association with greater availability of foods to populations in middle and high income countries. However, these foods are high in energy and fat but poor in nutrient density, resulting in a decrease in the consumption of omega-3 fatty acids, vitamins, flavonoids, as well as in essential and nonessential amino acids, significant increase in the intakes of carbohydrates, (mainly refined), fat (saturated, trans fat and linoleic acid) and salt compared to the early peasants in Paleolithic times (Tables 1 and 2). The protein or amino acid intake was 2.5 fold greater (33\% vs. 13\%) in the Paleolithic diet of Homo sapiens compared to modern Western diet consumed by Homo economicus populations. It has been estimated that diets of Homo sapiens were characterized by higher intakes of essential and non-essential amino acids, calcium, potassium, magnesium, flavonoids and w-3 fatty acids whereas the modern Western diet of Homo economicus has excess of energy-rich refined carbohydrates, w-6, trans fat and saturated fat and low in protective nutrients (Table 1 and 2). There has been marked changes in food and nutrient intakes during the last 100-160 years causing increased intake of saturated fatty acids (SFA), trans fatty acids, linoleic acid, and meat from grain-fed cattle, domesticated at farm houses, rather than meat from hunted animals $[2,3]$.

These differences in social class may be associated with differences in the food and nutrient intake among peasants and among Western and Asian populations during transition from poverty to affluence (Tables 1 and 2) [1-3,16,17]. Western type foods consumed by lower social classes in the developed countries and higher social classes in developing countries may cause marked reductions in the consumption of anti-atherogenic protective foods and significant increases in the intakes of pro-atherogenic foods (Tables 1 and 2). These foods in conjunction with sedentary behavior, mental strain, pollution, tobacco consumption and alcoholism, may have caused damage to our epigenome, leading to emergence of phenotypes of CVDs and other chronic diseases. Epigenetic damage can alter biological function in the offspring without causing changes in gene sequence. The higher social classes 1-3, who became victims of food security during transition 
from poverty to affluence, need functional food security. However, lower social classes 4 and 5 , need food security as well as functional food security, so that they do not suffer from NCDs.

\section{Thailand Declaration for Functional Food Security}

This declaration is important to educate world policy makers that functional food security is a step forward ahead of food security. For JUST the sake of food security, we should not be supportive of providing high energy, rapidly absorbed foods which are known to cause obesity and metabolic syndrome [1-3]. Food security may be defined as an increase in food availability to an extent, or by a policy, by through which food is available to poor, at affordable cost to prevent hunger and undernutrition and related diseases [16,17]. In the last few decades, in many developing and middle income countries, food security provided by the governments, in one sense understandably, gave least consideration to functional foods supply and the prevention of obesity and metabolic syndrome, resulting in rapid emergence of NCDs in these countries [1-3].

The Thailand Declaration reiterates that commitments to eradicate hunger and all forms of malnutrition including over-nutrition, and to increase investments in effective interventions; designers foods and designers crops [6-9], This should be executed urgently However, in planning coherent policies, our past experience on rapidly absorbed energy-rich processed foods should be taken in to account, while developing sustainable food systems. The food industry should be educated to exploit the expertise of food scientists and health professionals in designing functional foods taking cognizance of manufacturing and processing. Similarly, agriculture scientists may be actively involved in educating farmers so as to grow functional crops. In fact policy makers could provide sustained economic models for farmers to integrate land utilization for tourism, high cash crops for industry, functional foods including specialist crops and horticultural products The Thailand Declaration also agrees with Rome Declaration which have been agreed in advance but sustainable diets and food systems need further attention in view of the review of civil society organization [6]. It seems that our 4F agenda (FUNCTIONAL FOODS \& FUNCTIONAL FARMING) needs further attention on food chemistry and soil chemistry to provide functional farming, by the world experts.

Functional foods may be defined as those foods which are rich in certain nutrients that can address any physiological mechanisms while providing benefit to body systems, q.v. introduction. Functional food security means that such foods should be available at affordable cost for human consumption because of their role in providing optimal health and in the prevention of obesity and metabolic syndrome that are risk factors of NCDs [2,12,13,16-28]. It is important to plan for current and future needs taking into consideration healthcare costs which future generations may not be able to afford if functional food security is not sought. However, it is also important to educate people to understand the value of health education because knowledge of health foods and attitude towards these foods are also important in enhancing the consumption of such foods, with associated prevention strategies.

The latest estimates of FAO indicate that, $90 \%$ of the world's food supply is from $17 \%$ of plant species, which is mainly contributed by grains produced by fertilizer-based rapidly grown crops. Wheat, corn and rice account for three fourths of the world's grain production on which humans are dependent for their food supply [6-9]. Grains are high in omega-6 fatty acids and carbohydrates and low in omega-3 fatty acids and antioxidants compared to leafy green vegetables. Green leafy vegetables are also rich sources of antioxidants, magnesium, w-3 fatty acids, essential and nonessential amino acids and carotenoids which appear to be high in the Mediterranean region [1-5] e.g. Crete. There are fundamental contributions of agriculture to human nutrition through production, pricing and incomes. However, agriculture and the broader food system, including post-harvest processing and distribution and retailing, can contribute much more in providing better health options to populations. Food systems as a whole, from production through consumption, can be made 
more nutrition-enhancing and more environmentally sustainable by a number of specific actions that are identified in the report [7-9,17]. There is a need to actively promote the collaboration of all relevant stakeholders viz. health care professionals, food and agricultural scientists, to make a concerted effort to close the gaps that remain regarding information on basic data on diets, foods and on nutritional status and development of wellness or diseases.

Functional food security recognizes the potential of food systems to become both more sustainable and more supportive of good nutritional outcomes and the need for a multi-sectoral approach that includes agriculture and food systems, health, sanitation, social protection, employment and education. Further studies are needed for the evaluation of agricultural food-based interventions on nutritional outcomes; and to also encourage management practices and technologies to improve sustainability and nutrition. There is a need to evaluate the impacts of markets, trade and market structure on environmental sustainability and human nutrition; and recognize the roles of consumer choice in achieving objectives of optimal nutrition and sustainability $[7-9,12,17,20]$.

\section{Functional Foods in the Prevention of Diseases}

It is known that the Western diet has adverse effects; oriental foods are neutral, whereas Mediterranean-style foods are protective against NCDs [2,22-33]. The protective effects of Paleolithic diets in the form of the Mediterranean diet, Indo-Mediterranean diet, Japanese diet, and DASH diet have been documented in several previous studies [2,20-27]. It is expected that if, these diets are adopted by Homo economicus, and as such these standards will help evolve Homo modestis, either directly or by epigenetic inheritance or by natural selection [2]. Therefore, it is food security of functional foods, rather than modern western foods, which appear to be the important consideration for evolving human wellness and for prevention of NCDs.

A recent study showed that the consumption of total functional foods as well as fruits; grapes, apple, guava, star gooseberry, vegetables and nuts were strongly and inversely associated with risk of death due to NCDs [29]. The study also found that, Western type foods (red meat and eggs and refined foods) were positively associated with these causes of deaths, in both sexes. Total spices intake, mustard/olive oil intake and curd or yogurt intake, were inversely but weakly associated with causes of deaths due to NCDs, among both men and women [29]. Fruits and vegetables contain, over 600 carotenoid compounds (fat-soluble plant pigments), including lycopene, a-tocopherol, lutein, b-cryptoxanthin, zeaxanthin and beta-carotene $[2,19,21-24,26,28,29]$. Of these carotenoids, beta-carotene is the most widely studied which is the major vitamin A precursor and is carried in plasma and LDL particles. Carotenoids prevent lipid peroxidation by providing electrons to quench singlet oxygen. Lycopene is a potent antioxidant, the substance that gives tomatoes their red color, appears to be stronger than beta-carotene. Epidemiologic studies have correlated high tissue concentrations of lycopene with a lower risk of myocardial infarction; however, prospective, controlled trials have not yet been completed with lycopene. Tomato seeds also provide some flavanols in the tomato seed oil, which have antiplatelet effects.

\section{Clinical Evidence on Diet and NCDs.}

Clinical evidence on the role of Mediterranean diets as functional food is very strong because, such diets are known to reduce all-cause mortality. The EPIC-NL Study is a cohort of 40,011 subjects with a follow-up of 10-15 years [31]. The Mediterranean diet included daily intakes of vegetables, fruits, legumes and nuts, grains, fish, fatty acids, meat, dairy, and alcohol (wine and beer). Among 34,708 participants free of CVD at baseline, 4881 CVD events occurred, and 487 persons died from CVD. Increased intake of Mediterranean 
diet by two-units (range 0-9) was inversely associated with fatal CVD (HR: 0.78; 95\% CI: 0.69-0.88), total CVD (HR: 0.95 (0.91-0.98)), myocardial infarction, stroke and pulmonary embolism[31]. It is clear that a better adherence to a Mediterranean-style diet was more strongly associated with fatal CVD than with total CVD. The associations were strongest for incident myocardial infarction, stroke and pulmonary embolism. The relation between fruit and vegetable intake and incidence of CAD was examined in a meta-analysis among 278 459 subjects with 9143 CAD events during a median follow-up of 11 years. Among subjects consuming more than 5 servings/day of fruit and vegetables, the pooled RR of CAD was significantly greater compared with those consuming less than 3 servings/day, ( $P=0.0001$ vs 0.06).This meta-analysis reported that increased consumption of fruit and vegetables, from less than 3 to more than 5 servings/day is related to a $17 \%$ reduction, whereas increased intake to $3-5$ servings/day is associated with a smaller and borderline significant reduction in CAD risk. These results provide strong evidence supporting that 5 or more servings per day of fruit and vegetables can provide substantial protection from CVDs.

A large cohort study, involving 72,113 female nurses who were free of CAD, stroke, diabetes, and cancer, examined the association of dietary pattern with risk of NCDs [33]. During a follow-up of 18 years, 6011 deaths occurred (3319 (52\%) as a result of cancer; 1154 (19\%) resulting from CVDs; and 1718 (29\%) resulting from other causes. There was a $17 \%$ lower risk of total mortality among those who were most adherent to the prudent diet (highest versus lowest quintile of adherence), a 28\% lower risk of CVD mortality, and $30 \%$ lower mortality from non-CVD, non-cancer causes. Cancer was not associated with the inverse prudent dietary pattern. A comparison of the highest and lowest quintiles of adherence showed that consumption of the Western diet was associated with increased total mortality (21\%), CVD mortality (22\%), cancer mortality (16\%), and mortality from non-CVD, non-cancer causes (31\%). Hence, except for cancer, risk relationships for the prudent and Western dietary patterns appear to be the inverse of each other: mortality thus was increased as adherence to the prudent diet decreased and adherence to the Western diet increased.

The INTERHEART study, ( $\mathrm{n}=\mathrm{ACS}=5761$, controls=10,646) examined the relationship between dietary patterns and risk of acute coronary syndrome (ACS) [32]. The investigators identified 3 major dietary patterns viz. Oriental (high intake of tofu and soy and other sauces), Western (high in fried foods, salty snacks, eggs, and meat), and prudent (high in fruit and vegetables). Consistent with previous studies in single within-population cohort studies, the authors found an inverse association between the prudent pattern score and risk of ACS and a significant positive association between the Western pattern score and increased risk of ACS. No association of Oriental diet with risk of ACS was reported. A dietary risk score based on 7 food items on the food-frequency questionnaire (meat, salty snacks, fried foods, fruits, green leafy vegetables, cooked vegetables, and other raw vegetables) was constructed by the authors. Further sensitivity analyses revealed a consistent association for the composite diet score between men and women and across different regions of the world (North America, Western Europe, Australia, Central Europe, Middle East, Africa, south Asia, Southeast Asia, China, and South America). On the basis of an arbitrary cut point of the score (top 3 quartiles versus the bottom quartile), the investigators estimated that $30 \%$ of myocardial infarction (MI) could be explained by unhealthy diets worldwide. The benefits of prudent dietary patterns in the INTERHAERT Study appear to be due increased intake of fruits, vegetables, and whole grains which are rich sources of functional nutrients.

The effects of functional foods for CAD risk reduction in a meta-analysis showed that these foods are protective against $\mathrm{CAD}$ [34].In another systematic review of studies, which included the results from 14 human studies (7 prospective, 3 cross-sectional, 1 controlled, 3 case-control) and 13 animal studies, found that dietary omega-6 to omega-3 fatty acid ratio can influence brain composition[35], Alzheimer's disease pathology, and behavior (as according to animal studies), and revealed an association between the omega- 6 to omega-3 ratio, cognitive decline, and incidence of dementia. This review supports growing evidence of a positive association 
between the dietary omega-6/omega-3 ratio and the risk of Alzheimer's disease. In the NHANES 2003-2008, high intake of dietary long-chain $\omega-3$ fatty acids was associated with lower blood pressure in children born with low birth weight [36].

In a randomized, controlled intervention trial, Singh et al examined the effects of guava intake on serum total and high density lipoprotein cholesterol, lipid peroxides levels and systemic blood pressures among 120 patients with hypertension for 3 months[37]. In this study, 61 group A and 59 group B patients with essential hypertension were administered guava fruit preferably before meals in a foods-to-eat approach rather than foods-to-restrict, in a randomized and single-blind fashion for 12 weeks [37]. Nutrient intakes including saturated and total fat were significantly decreased; carbohydrates, total and soluble fiber, flavonoids and vitamins and mineral intakes were significantly higher in group A than in group B at 12 weeks. There was a significant net decrease in serum total cholesterol (9.9\%), triglycerides $(7.7 \%)$ and blood pressures $(9.0 / 8.0 \mathrm{~mm}$ $\mathrm{Hg}$ ) with a significant net increase in high-density lipoprotein cholesterol (8.0\%) after 12 weeks of guava fruit substitution in group A than in group B. By adding moderate amounts of guava fruit in the usual diet, changes in dietary fatty acids and carbohydrates may occur, providing significant amounts of total and soluble dietary fiber and antioxidant vitamin C, potassium, flavonoids and minerals which are known to provide, lipid lowering, antihypertensive and antioxidant cardio-protective effects, without any adverse effects.

Effects of fat modified and fruits vegetable enriched diets on blood lipids and lipid peroxides in the Indian diet heart study among 621 high risk subjects revealed that diet can decrease blood lipids, blood glucose and oxidative stress and increase high density lipoprotein cholesterol[9]. In a randomized, single blind controlled trial with functional food rich diet among 406 patients of acute coronary syndromes, beneficial effects were noted within six weeks [22] and after one year of follow up [23]. The intervention group received significantly greater amount of functional foods; fruits; apple, grapes, guava, papaya, oranges, vegetables; bitter gourds, green leafy vegetables, cabbage, brinjal, carrots, ladyfingers, onion, garlic, ginger, nuts; almonds, walnuts in conjunction with 20-30 g/day of mustard oil compared to control group.

The Lyon diet heart study including Mediterranean-style diet and rapeseed oil margarine, given to 600 post heart attack patients and the Indo-Mediterranean diet heart study including fruits, vegetables and mustard oil, given to 1000 high risk subjects, also reported beneficial effects of functional food rich diets, (low in omega-6/omega-3 fatty acids) on morbidity and mortality among patients with high risk of CVDs[2,24,27,28]. The beneficial effects of this diet were attributed to decreased omega-6/omega-3 fatty acid ratio in the diets $[2,19,27,28]$. Anatomy of health effects of Mediterranean diets in the Greek epic prospective heart study also revealed cardio-protective effects of this diet[26]. The PREDIMED trial enrolled a total of 7,447 persons, aged 55 to 80 years including $57 \%$ women who were followed up for 4.8 years[38]. A primary end-point event occurred in 288 participants. The multivariable-adjusted hazard ratios were 0.70 (95\% confidence interval CI, 0.54 to 0.92$)$ and 0.72 (95\% CI, 0.54 to 0.96 ) for the group assigned to a Mediterranean diet with extra-virgin olive oil and the group assigned to a Mediterranean diet with nuts, respectively, versus the control group (96 and 83 vs 109 events). No diet-related adverse effects were reported. Among persons at high cardiovascular risk, a Mediterranean diet supplemented with extra-virgin olive oil or nuts reduced the incidence of major cardiovascular events in both intervention groups compared to control group.

The Sydney Diet Heart Study included 458 men aged 30-59 years with a recent coronary event [39]. Inclusion of these recovered data in an updated meta-analysis of linoleic acid intervention trials showed non-significant trends toward increased risks of death from CAD (hazard ratio 1.33 (0.99 to 1.79); $\mathrm{P}=0.06$ ) and CVDs (1.27 (0.98 to 1.65); $\mathrm{P}=0.07$ ) indicating that substituting dietary linoleic acid in place of saturated fats increased the rates of death from all causes, as well as CAD, and CVD. 
Mustard oil and fish oil are also functional foods due to high content of w-3 fatty acids hence both are cardio-protective [23]. Randomized, double-blind, placebo-controlled trial of fish oil and mustard oil in 360 patients with suspected acute myocardial infarction revealed that eating fish oil or mustard oil can cause significant decline in morbidity and mortality during a follow up of one year[23]. Apart from vegetable, fruits, nuts and pulses, cocoa in foods such as dark chocolates, green and black tea flavonols, probiotics and leafy vegetables which are rich in polyphenolics are other functional foods [18,40-45].

These findings indicate that this approach appears to be useful for the food industry to develop functional foods by further modifications of probiotics with omega-3 fatty acids, flavanols by adding cocoa, walnuts and black raisins, etc, based on well-conducted clinical studies for clearly identified populations.

\section{Necessity of Biotechnology for Food Production}

There may be substantial improvement in food production qualitatively as well as quantitatively due to use of biotechnology [46-48]. Plant breeding and genetic modifications are latest technologies for developing diversity in foods by altering nutrient content of foods to solve the problem of functional food security [4,46-49]. Agriculture played a vital role during the era of green revolution initiated by Norman Borlaug, the "Father of the Green Revolution" who saved over a billion people from starvation and developed high-yielding varieties of cereal grains. However, these food varieties were high in energy and deficient in micronutrients which are known to provide wellness and reduced risk of NCDs. I

It seems that the use of biotechnology has also upraised concerns about its potential risks to the environment as well as to human beings, resulting in emergence of obesity and metabolic syndrome. The staple crops can be enriched with micronutrients using plant breeding strategies. Now at this point of time, agriculture can again play a vital role by developing food varieties high in micronutrients, macronutrients, organic acids, trace mineral, antioxidants, flavonoids and vitamins leading to functional food security. The traits responsible for micronutrient enrichment in crops exist in their wild types or can be said that these traits are present within their genomes which can be used for substantially increasing micronutrient levels in crops without any negative impact on crop productivity [46-48]. The fruits and vegetables, which are consumed raw, can be subjected for improvement of micronutrients through application of new technologies of plant breeding and genetic engineering.

Genetic Engineering provides resources to host genes into plants via genetic mechanisms, different in some respects from classical breeding. A number of genetically engineered variety of foods have been developed, which have become important nutraceuticals; most notably canola, cotton, maize and soybean, were developed employing this modern technology, and at present the traits introduced are herbicide and/or pest tolerance. Gene transfer technology is employed to alter the physical and chemical composition with worthy nutraceutical value $[46,49]$. This technology aims largely to increase the production in the species, as well as the elevation of resistance to pests, viruses, frost, etc. Genetic modification of food (GMF) may be useful in many countries with vitamin A deficiency causing large numbers of vision-impaired people and increased mortality due to a weakened immune system [4]. The Golden Rice Project is being moved forward at various levels and the most advanced version of Golden Rice, which was produced by Syngenta scientists, has been coined GR2 [4]. This improved version produces $31 \mu \mathrm{g} / \mathrm{g}$ and more $\beta$-carotene, which is more than enough to supply the required amounts of $\beta$-carotene, according to the bioavailability results. Golden Rice 2", introducing this variety in combination with the Erwinia uredovora carotene desaturase (CRTI) used to generate the original Golden Rice, has also been developed[4]. There is an increase in total carotenoids of up to 23-fold (maximum $37 \mu \mathrm{g} / \mathrm{g}$ ) compared to the original Golden Rice and a preferential accumulation of beta-carotene. Bohn et al using 35 different nutritional and elemental variables to characterize each soy sample were able to 
discriminate GM, conventional and organic soybeans without exception [49]. They demonstrated "substantial non-equivalence" in compositional characteristics for 'ready-to-market soy samples. Organic soybeans showed the healthiest nutritional profile with more sugars, such as glucose, fructose, sucrose and maltose, significantly more total protein, zinc and less fiber than both conventional and GM-soy. Organic soybeans also contained less total saturated fat and total omega- 6 fatty acids than both conventional and GM-soy. GM-soy contained high residues of glyphosate and AMPA (mean 3.3 and $5.7 \mathrm{mg} / \mathrm{kg}$, respectively). Conventional and organic soybean batches contained none of these agrochemicals. There may be several obstacles toward a healthy lifespan, both political and financial related foods and farming. The sustainable development is of vital importance but require resource mobilization, measurable indicators, clarity, honesty and accountability. Prevention at population level, health equity, and the right to health would be truly transformative, if the functional food security using GM foods is used honestly[50].

"You are what you eat" was proposed by Hippocrates in the 5th century BC. Hippocrates wrote "Positive health requires knowledge of man's primary constitution (which today we call genetics) and of the powers of various foods, both those natural to them and those resulting from human skill (today's processed foods which should be rich in nutrients and slowly absorbed).

\section{Conclusions}

In brief, functional foods are rich in protective nutrients; omega-3 fatty acids, monounsaturated fatty acids, flavonoids, amino acids, vitamins, antioxidants and fiber with low energy density. Biotechnology including food processing and plant breeding or genetic engineering, may be used to increase protective nutrients; and reduce too much of certain nutrients causing adverse effects, (saturated fat, linoleic acid, sugar and erucic acid). In future, every attempt should be made to develop protective nutrient rich, low energy, slowly absorbed functional foods to provide functional food security, for control of under-nutrition so that there is no increase in obesity which is important for prevention of CVDs and other chronic diseases.

Conflict of interest: The authors declare that there is no conflict of interest regarding the publication of this article

\section{References}

1. Singh, R.B.; Takahashi, T.; Nakaoka, T.; Otsuka, K.; Toda, E.; Shin, H.H.; Lee, M.-K.; Beeharry, V.; Hristova, K.; Fedacko, J., et al. Nutrition in transition from homo sapiens to homo economicus. The Open Nutraceuticals Journal 2013, 6, 6-17.

2. De Meester, F. Progress in lipid nutrition: The columbus concept addressing chronic diseases. In A balanced omega-6/omega-3 fatty acid ratio, cholesterol and coronary heart disease, A.P., S.; F., D.M., Eds. World Review of Nutrition and Dietetics: Karger AG, Basel, 2009; Vol. 100, pp 110-121.

3. Lindeberg, S. Food and western disease: Health and nutrition from an evolutionary perspective. Wiley-Blackwell: UK, 2010.

4. $\quad$ Paine, J.A.; Shipton, C.A.; Chaggar, S.; Howells, R.M.; Kennedy, M.J.; Vernon, G.; Wright, S.Y.; Hinchliffe, E.; Adams, J.L.; Silverstone, A.L., et al. Improving the nutritional value of golden rice through increased pro-vitamin a content. Nat Biotech 2005, 23, 482-487.

5. 18th World Congress on Clinical Nutrition; Agriculture, F.a.N.a.W. Abstract Ubonratchathani University Thailand 2014.

6. Lancet, T. Feeding the world sustainably. The Lancet 2014, 384, 1721. 
7. UNICEF. Child nutrition interactive dashboard. http://data.unicef.org/resources/2013/webapps/nutrition

8. WHO. Unicef-who-the world bank: Joint child malnutrition estimates - levels and trends.

9. UNICEF-WHO. The world bank: Joint child malnutrition estimates - levels and trends. http://www.who.int/nutgrowthdb/estimates/en/

10. Lancet. Feeding the world sustainably. The Lancet 2014, 384, 1721.

11. Hristova, K.; Shiue, I.; Pella, D.; Singh, R.B.; Chaves, H.; Basu, T.K.; Ozimek, L.; Rastogi, S.S.; Takahashi, T.; Wilson, D., et al. Prevention strategies for cardiovascular diseases and diabetes mellitus in developing countries: World conference of clinical nutrition 2013. Nutrition 2014, 30, 1085-1089.

12. Singh, R.B.; Reddy, K.K.; Meester, F.D.; Wilczynska, A.; Wilson, D.W. Ancient concepts in nutrition and diets in hunter-gatherers. The Open Nutraceuticals Journal 2011, 4, 130-135.

13. Eaton, S.B.; Konner, M.; Shostak, M. Stone agers in the fast lane: Chronic degenerative diseases in evolutionary perspective. The American Journal of Medicine 1988, 84, 739-749.

14. Eaton, S.B.; Konner, M. Paleolithic nutrition. New England Journal of Medicine 1985, 312, $283-289$.

15. Meester, F. Wild-type land-based food in health promotion and disease prevention. In Wild-type food in health promotion and disease prevention: The columbus concept, Meester, F.; Watson, R.R., Eds. Humana Press: Totowa, NJ, 2008; pp 3-20.

16. Popkin, B.M. Global nutrition dynamics: The world is shifting rapidly toward a diet linked with noncommunicable diseases. The American journal of clinical nutrition 2006, 84, 289-298.

17. FAO. The state of food and agriculture social protection and agriculture: Breaking the cycle of rural poverty. http://www.fao.org/publications/sofa/2015/en/

18. Hristova, K.; Shiue, I.; Pella, D.; Singh, R.B.; Chaves, H.; Basu, T.K.; Ozimek, L.; Rastogi, S.S.; Takahashi, T.; Wilson, D., et al. Prevention strategies for cardiovascular diseases and diabetes mellitus in developing countries: World conference of clinical nutrition 2013. Nutrition 2013, 30, 1085-1089.

19. Simopoulos, A.P. Evolutionary aspects of the dietary omega-6:Omega-3 fatty acid ratio: Medical implications. In A balanced omega-6/omega-3 fatty acid ratio, cholesterol and coronary heart disease, Simopoulos, A.P.; De Meester, F., Eds. World Review of Nutrition and Dietetics: Karger AG, Basel, 2009; Vol. 100, pp 1-21.

20. Hristova, K.; Nakaoka, T.; Otsuka, K.; Fedacko, J.; Singh, R.; Singh, R.B.; Meester, F.D.; Wilczynska, A.; Wilson, D.W. Perspectives on chocolate consumption and risk of cardiovascular dis-eases and cognitive function. The Open Nutraceuticals Journal 2012, 5, 207-212.

21. Singh, R.B.; Rastogi, S.S.; Niaz, M.A.; Ghosh, S.; Singh, R.; Gupta, S. Effect of fat-modified and fruitand vegetable-enriched diets on blood lipids in the indian diet heart study. The American journal of cardiology 1992, 70, 869-874.

22. Singh, R.B.; Rastogi, S.S.; Verma, R.; Bolaki, L.; Singh, R. An indian experiment with nutritional modulation in acute myocardial infarction. The American journal of cardiology 1992, 69, 879-885.

23. Singh, R.B.; Rastogi, S.S.; Verma, R.; Laxmi, B.; Singh, R.; Ghosh, S.; Niaz, M.A. Randomised controlled trial of cardioprotective diet in patients with recent acute myocardial infarction: Results of one year follow up. BMJ : British Medical Journal 1992, 304, 1015-1019.

24. Singh, R.B.; Dubnov, G.; Niaz, M.A.; Ghosh, S.; Singh, R.; Rastogi, S.S.; Manor, O.; Pella, D.; Berry, E.M. Effect of an indo-mediterranean diet on progression of coronary artery disease in high risk patients (indo-mediterranean diet heart study): A randomised single-blind trial. The Lancet 2002, 360, 1455-1461. 
25. de Lorgeril, M.; Renaud, S.; Salen, P.; Monjaud, I.; Mamelle, N.; Martin, J.L.; Guidollet, J.; Touboul, P.; Delaye, J. Mediterranean alpha-linolenic acid-rich diet in secondary prevention of coronary heart disease. The Lancet 1994, 343, 1454-1459.

26. Trichopoulou, A.; Bamia, C.; Trichopoulos, D. Anatomy of health effects of mediterranean diet: Greek epic prospective cohort study. BMJ 2009, 338.

27. Singh, R.B.; Fedacko, J.; Vargova, V.; Pella, D.; Niaz, M.A.; Ghosh, S. Effect of low w-6/w-3 fatty acid ratio paleolithic style diet in patients with acute coronary syndromes: A randomized, single blind, controlled trial. World Heart Journal 2012, 4, 71-84.

28. Pella, D.; Dubnov, G.; Singh, R.B.; Sharma, R.; Berry, E.M.; Manor, O. Effects of an indo-mediterranean diet on the omega-6/omega-3 ratio in patients at high risk of coronary artery disease: The indian paradox. In Omega-6/omega-3 essential fatty acid ratio: The scientific evidence Simopoulos, A.P.; Cleland, L.G., Eds. Karger Basel, 2003; pp 74-80.

29. Singh, R.B.; Visen, P.; Sharma, D.; Sharma, S.; Mondo, R.; Sharma, J.P.; Sharma, M.; Tokunaga, M.; Takahashi, T.; Mishra, S., et al. Study of functional foods consumption patterns among decedents dying due to various causes of death. The Open Nutraceuticals Journal 2015, 8, 16-28.

30. He, F.J.; Nowson, C.A.; Lucas, M.; MacGregor, G.A. Increased consumption of fruit and vegetables is related to a reduced risk of coronary heart disease: Meta-analysis of cohort studies. Journal of human hypertension 2007, 21, 717-728.

31. Hoevenaar-Blom, M.P.; Nooyens, A.C.; Kromhout, D.; Spijkerman, A.M.; Beulens, J.W.; van der Schouw, Y.T.; Bueno-de-Mesquita, B.; Verschuren, W.M. Mediterranean style diet and 12-year incidence of cardiovascular diseases: The epic-nl cohort study. PloS one 2012, 7, e45458. Iqbal, R.; Anand, S.; Ounpuu, S.; Islam, S.; Zhang, X.; Rangarajan, S.; Chifamba, J.; Al-Hinai, A.; Keltai, M.; Yusuf, S. Dietary patterns and the risk of acute myocardial infarction in 52 countries: Results of the interheart study. Circulation 2008, 118, 1929-1937.

33. Heidemann, C.; Schulze, M.B.; Franco, O.H.; van Dam, R.M.; Mantzoros, C.S.; Hu, F.B. Dietary patterns and risk of mortality from cardiovascular disease, cancer, and all causes in a prospective cohort of women. Circulation 2008, 118, 230-237.

34. Castro, I.A.; Barroso, L.P.; Sinnecker, P. Functional foods for coronary heart disease risk reduction: A meta-analysis using a multivariate approach. The American journal of clinical nutrition 2005, 82, 32-40.

35. Loef, M.; Walach, H. The omega-6/omega-3 ratio and dementia or cognitive decline: A systematic review on human studies and biological evidence. Journal of nutrition in gerontology and geriatrics 2013, 32, 1-23.

36. Skilton, M.R.; Raitakari, O.T.; Celermajer, D.S. High intake of dietary long-chain omega-3 fatty acids is associated with lower blood pressure in children born with low birth weight: Nhanes 2003-2008. Hypertension 2013, 61, 972-976.

37. Singh, R.B.; Rastogi, S.S.; Singh, R.; Ghosh, S.; Niaz, M.A. Effects of guava intake on serum total and high-density lipoprotein cholesterol levels and on systemic blood pressure. The American journal of cardiology 1992, 70, 1287-1291.

38. Estruch, R.; Ros, E.; Salas-Salvadó, J.; Covas, M.-I.; Corella, D.; Arós, F.; Gómez-Gracia, E.; Ruiz-Gutiérrez, V.; Fiol, M.; Lapetra, J., et al. Primary prevention of cardiovascular disease with a mediterranean diet. New England Journal of Medicine 2013, 368, 1279-1290.

39. Ramsden, C.E.; Zamora, D.; Leelarthaepin, B.; Majchrzak-Hong, S.F.; Faurot, K.R.; Suchindran, C.M.; Ringel, A.; Davis, J.M.; Hibbeln, J.R. Use of dietary linoleic acid for secondary prevention of coronary 
heart disease and death: Evaluation of recovered data from the sydney diet heart study and updated meta-analysis. Bmj 2013, 346, e8707.

40. Barraud, D.; Bollaert, P.E.; Gibot, S. Impact of the administration of probiotics on mortality in critically ill adult patients: A meta-analysis of randomized controlled trials. Chest 2013, 143, 646-655.

41. Rochfort, S.; Panozzo, J. Phytochemicals for health, the role of pulses. Journal of Agricultural and Food Chemistry 2007, 55, 7981-7994.

42. Sreeramulu, D.; Reddy, C.V.; Raghunath, M. Antioxidant activity of commonly consumed cereals, millets, pulses and legumes in india. Indian journal of biochemistry \& biophysics 2009, 46, 112-115.

43. Velioglu, Y.S.; Mazza, G.; Gao, L.; Oomah, B.D. Antioxidant activity and total phenolics in selected fruits, vegetables, and grain products. Journal of Agricultural and Food Chemistry 1998, 46, 4113-4117.

44. Arcan, I.; Yemenicioğlu, A. Antioxidant activity and phenolic content of fresh and dry nuts with or without the seed coat. Journal of Food Composition and Analysis 2009, 22, 184-188.

45. Gupta, S.; Prakash, J. Studies on indian green leafy vegetables for their antioxidant activity. Plant Foods for Human Nutrition 2008, 64, 39-45.

46. Welch, R.M.; Graham, R.D. Breeding for micronutrients in staple food crops from a human nutrition perspective. Journal of experimental botany 2004, 55, 353-364.

47. Bouis, H.E. Enrichment of food staples through plant breeding: A new strategy for fighting micronutrient malnutrition. Nutrition 2000, 16, 701-704.

48. Mishra, S.N.; Singh, R.B. Physiological and biochemical significance of genetically modified foods: An overview. Open Nutraceuticals Journal 2013, 6, 18-26.

49. Bøhn, T.; Cuhra, M.; Traavik, T.; Sanden, M.; Fagan, J.; Primicerio, R. Compositional differences in soybeans on the market: Glyphosate accumulates in roundup ready gm soybeans. Food Chemistry 2014, 153, 207-215.

50. Gostin, L.O.; Friedman, E.A. The sustainable development goals: One-health in the world's development agenda. Jama 2015, 314, 2621-2622.

Table 1. Food and nutrient intake among various social groups

\begin{tabular}{|c|c|c|c|}
\hline Food and nutrient & Hunter-gatherer society & Western society & Asian societies \\
\hline Energy density & Low & High & Moderate \\
\hline Protein & High $(33 \%)$ & Low $(12-14 \%)$ & Low $(8-12 \%)$ \\
\hline Animal & High & Low-moderate & Low-moderate \\
\hline Vegetable & Very low & Low -moderate & Low-Moderate \\
\hline Carbohydrate & Low-moderate (slowly & Moderate-rapidly & High \\
\hline & absorbed) & absorbed & Slow \\
\hline Fiber & High $(>30 g)$ & Low $(<15 g)$ & Moderate \\
\hline Fat & Low & High & Low \\
\hline Animal & Low & High & Low \\
\hline Vegetable & Very low & Low & $0.5-0.85 \mathrm{~g} / \mathrm{d}$ \\
\hline Total w-3 & $\operatorname{High}(2.3 \mathrm{~g} /$ day $)$ & $(0.2 \mathrm{~g} / \mathrm{d})$ & $25-50$ \\
\hline Ratio w-6:w-3 & Low 2.4 & High $15-25$ & moderate \\
\hline Vitamins and minerals & High & Low & \\
\hline Polyphenolics and & & & Moderate \\
\hline Flavonoids & High & Low & \\
\hline
\end{tabular}


Modified from Eaton et al. [13,14] and Singh et al. [1] references.

Table 2. Nutrition in transition and emergence of non-communicable diseases

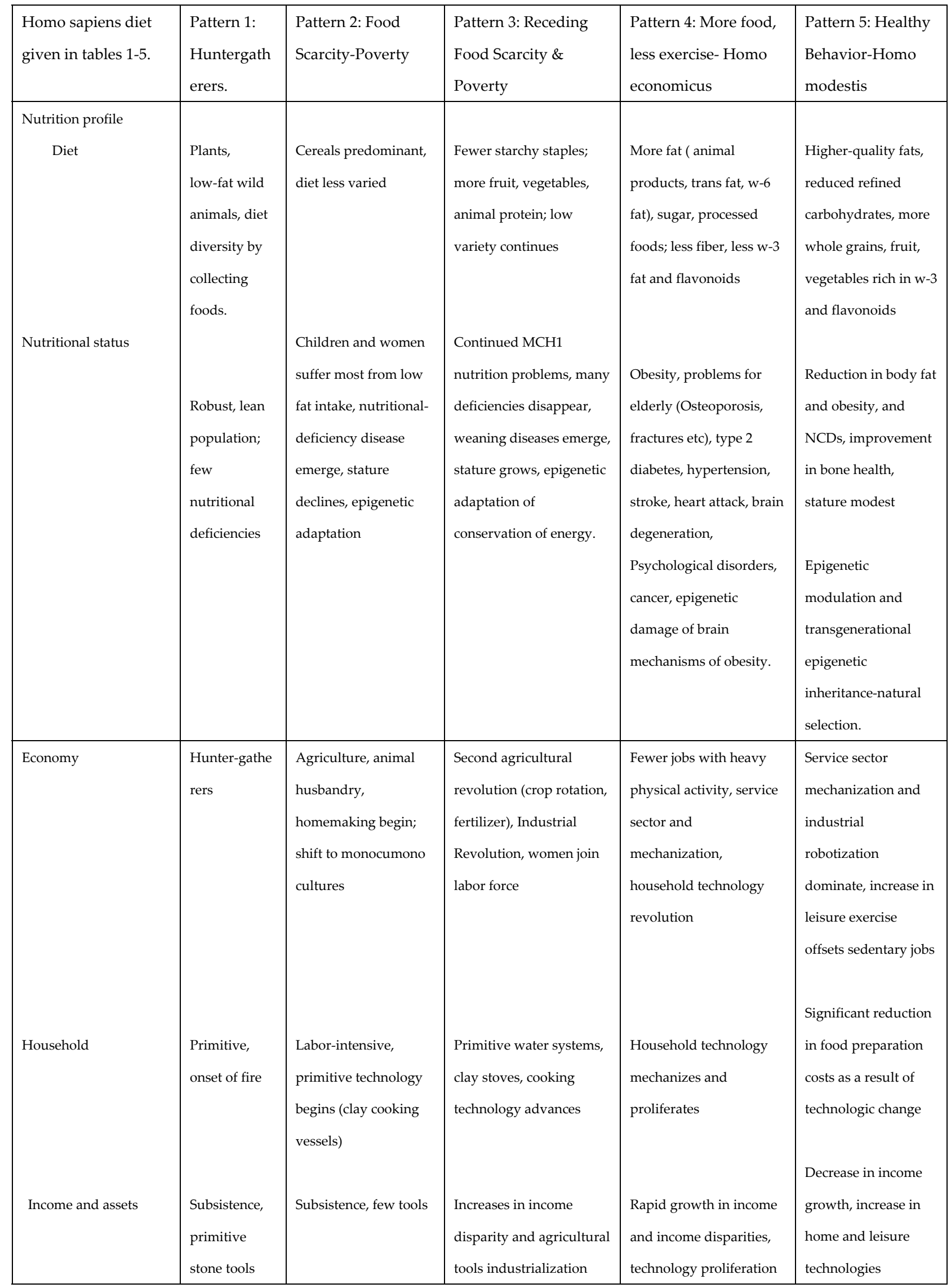




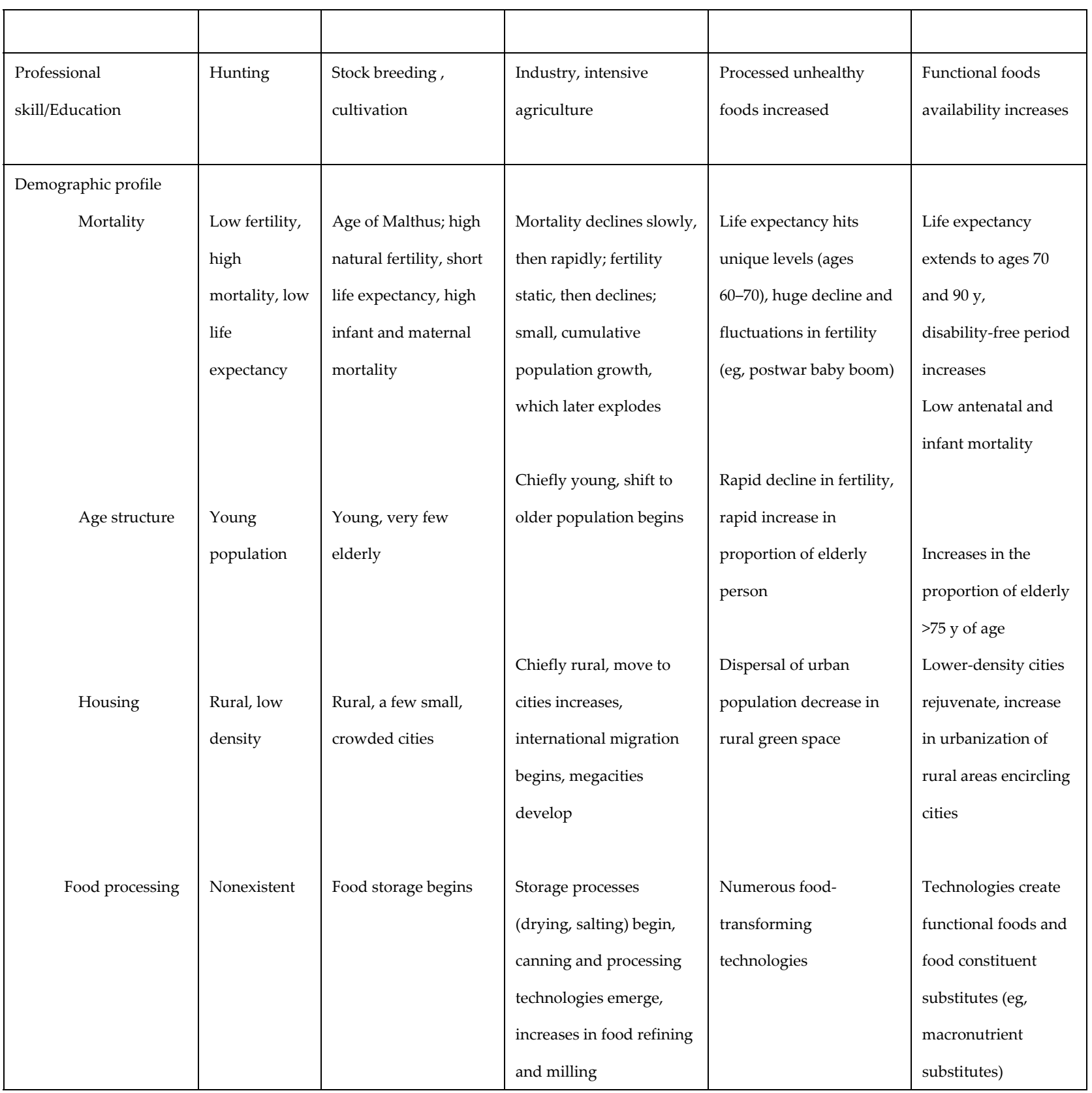

$1 \mathrm{MCH}$, maternal and child health, Modified from Popkin 2006, Singh et al 2013,References [1,15]. 